\title{
Concept of mobile lab for electric vehicle systems research and testing
}

\author{
Mario Vražić, Marinko Kovačić, Hrvoje Džapo, Damir llić \\ University of Zagreb Faculty of Electrical Engineering and Computing (FER), Unska 3, HR-10000 Zagreb
}

ABSTRACT

When research of different systems (measurement, control, etc.) on an electric vehicle is required, it is very useful to have some sort of a test bed for the system under investigation. In order to enable research of this kind, at the closest way possible to a real situation, the design and a large part of the actual realization was made on a test electric vehicle that should pass homologation and be able to drive on public roads. The vehicle with the systems required for the realization of the concept, as well as the possibilities of the future updates, will be presented in this paper.

Keywords: electric vehicle, measurement system, CAN bus communication

Citation: Mario Vražić, Marinko Kovačić, Hrvoje Džapo, Damir llić, "Concept of mobile lab for electric vehicle systems research and testing”, Acta IMEKO, vol. 2, no. 1, article 9, August 2013, identifier: IMEKO-ACTA-02(2013)-01-09

Editors: Paolo Carbone, University of Perugia, Italy; Ján Šaliga, Technical University of Košice, Slovakia; Dušan Agrež, University of Ljubljana, Slovenia

Received January $10^{\text {th }}, 2013$; In final form July $11^{\text {th }}, 2013$; Published August 2013

Copyright: ( 2013 IMEKO. This is an open-access article distributed under the terms of the Creative Commons Attribution 3.0 License, which permits unrestricted use, distribution, and reproduction in any medium, provided the original author and source are credited

Funding: none reported

Corresponding author: Mario Vražić, e-mail: mario.vrazic@fer.hr

\section{INTRODUCTION}

The main scope of this research is the development of a system that is capable of various measurements on the electric vehicle. The requirement for research on the electric vehicle is to test different types of the motor and battery configurations, thus the measurement system has to be universal and adaptable to fulfil this requirement. Different control systems as well as power conversion systems have to be considered.

There are several papers that are dealing with different topologies of the vehicle measurement system or with the individual components such as current or voltage measurements [1-3]. In their paper Ying et al. [4] report an implementation of the monitoring system based on the PC DAQ unit. Zhilong et al. describe a monitoring system built around a CAN-bus as the communication interface [5]. Weiss [6] describes an internal combustion vehicle conversion procedure and the precautions that must be taken into account.

It was decided that an old (cheap) but suitable car should be bought. The main reason for buying such a car was not its low price, but lack of modern technology and electric devices and aids. This particularly includes servo aids for brakes and for turning of the wheel. Without these aids there are no additional unnecessary loads for electric power supply and researchers can focus on the drive train instead providing power to the auxiliary systems of modern cars.

Also it is desirable that the car is equipped with rear wheel drive transmission and a longitudinally placed internal combustion engine. This enables easy exchange of different electric motors instead of the internal combustion engine. The gearbox is then placed outside the engine space (front vehicle

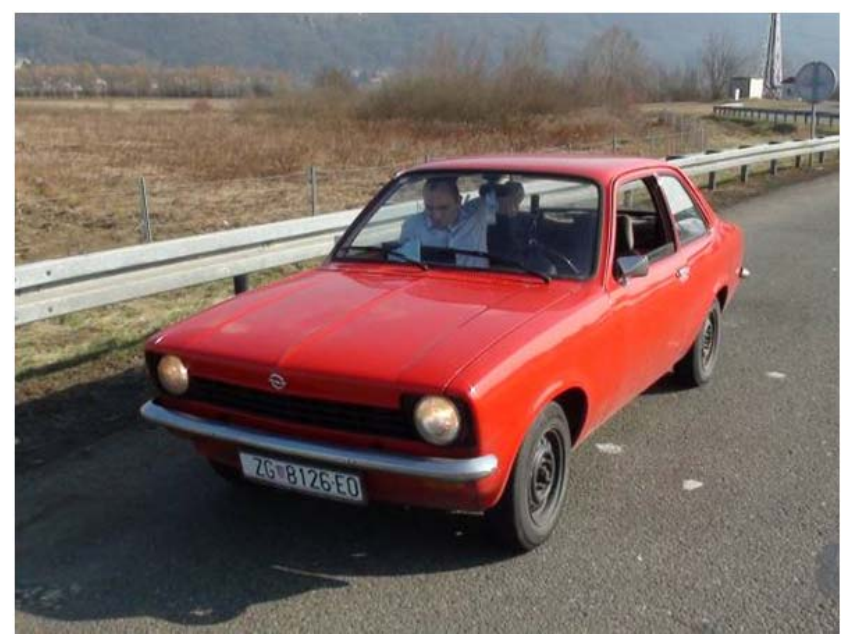

Figure 1. Opel Kadett C - test vehicle. 


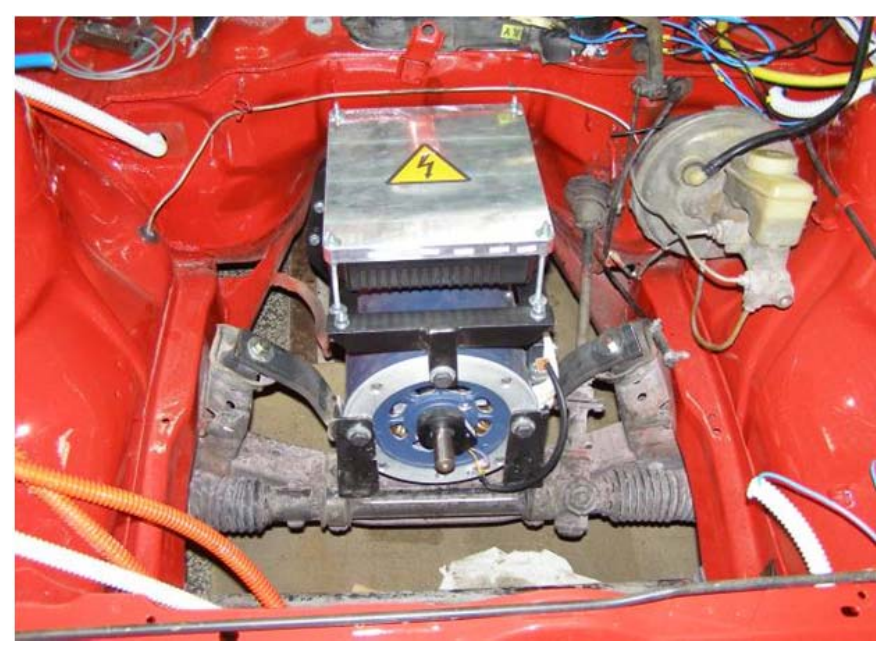

Figure 2. Induction motor with frequency converter installed in the vehicle.

volume) so this space is left available for additional equipment such as torque sensors, power converters, batteries, etc.

After a careful study of those requirements an oldtimer was chosen and purchased. As shown in Fig. 1 it is an Opel Kadett C from 1978. When buying a car of that age there are lots of precautions which have to be taken into account, the most important of them is the quality of the bodywork and the suspension. The main concern was its bodywork so it was carefully checked and was proven to be excellent.

The selected car has most of the requested features. Its weight is only $750 \mathrm{~kg}$, it has no servo for brakes nor for wheels, and it has rear transmission that includes a gearbox with 4 gears, a cardan shaft and a rear differential transmission. So its internal combustion engine could be replaced with an electric motor (Fig 2).

The electric motor is an induction motor with peak power of $37 \mathrm{~kW}$. A frequency converter has the ability of regenerative braking with the motor thus returning energy into the battery pack. The induction motor is installed onto gearbox (Fig. 3).

A battery pack with a nominal DC voltage of $102.4 \mathrm{~V}$, a capacity of $10.24 \mathrm{kWh}$ (or 100Ah), $\mathrm{LiFeMnPO}_{4}$ chemistry, and a weight of $100 \mathrm{~kg}$ is installed in the vehicle. The battery pack is divided into two equal parts. One is installed in the front part (in front of the motor - Fig. 4), and the other in the back. The total weight of the equipment installed in the car is practically the same as the parts which have been removed from the

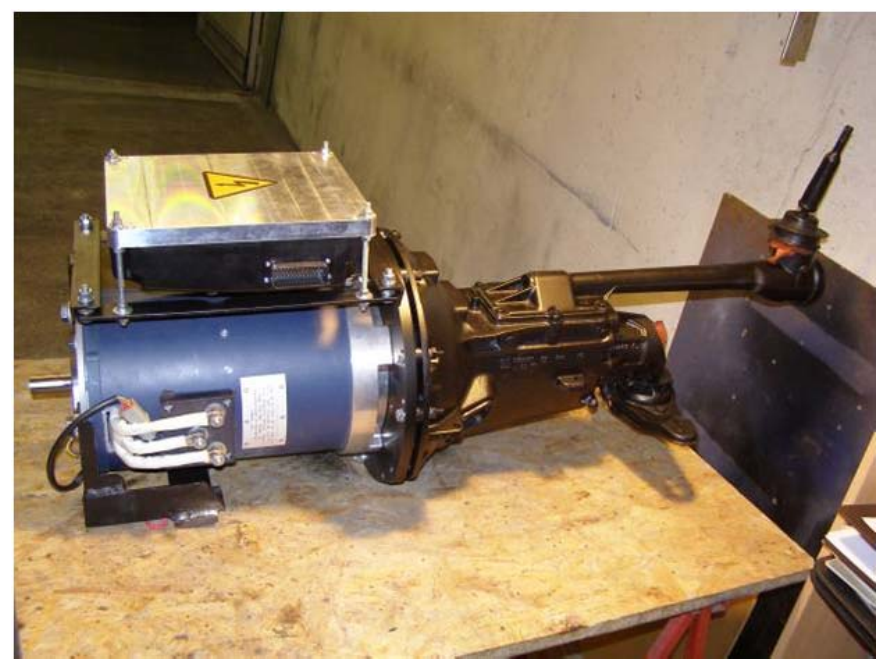

Figure 3. Induction motor with gearbox.

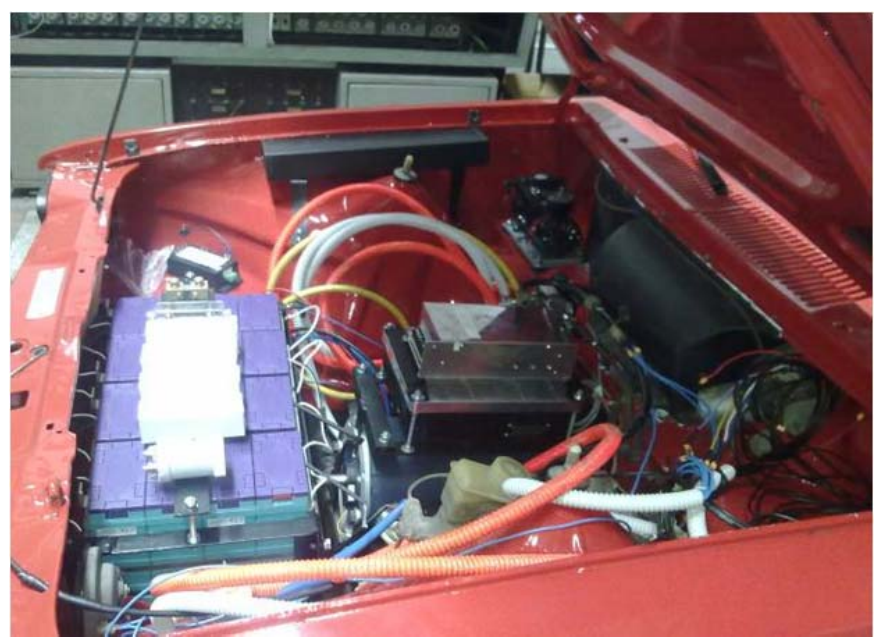

Figure 4. Battery pack in front of the induction motor.

vehicle (internal combustion engine, fuel tank, battery, cooler, all liquids, exhaust system, etc.). With the additional modification it is possible to add front wheel drives, preferably independent electric hub motors for each front wheel. This will enable research on the electric differential transmission as well as on the other electronically controlled features on vehicles such as regenerative braking.

Lots of other possibilities are open with this project but the primary goal was to design a measurement system capable to fulfil all requirements for this research. There are many research studies reported [7-10]. Before modification some measurements were done on the vehicle. The drive wheel torque and the rotational speed for different driving regimes have been measured. The noise level within the vehicle was measured at the same time as well.

\section{MEASUREMENT SYSTEM POSSIBILITIES}

During the design and development of this vehicle lots of different ideas and concepts were considered. One of them was to use a programmable logic controller (PLC) as a core of the measurement system as well as a control system (Fig. 5). Such a system has one major shortcoming due to the significant lack of memory suitable for long-term measurement data logging. Moreover, it is rather an expensive solution.

Another approach for the data acquisition system based on standard DAQ components is depicted in Fig. 6. This is a much better solution but it is also, due to current transducers and isolation amplifiers, a rather expensive system.

Finally, an idea that incorporates CAN communication as a backbone for the measurement system is proposed (Fig. 7). Namely a frequency converter for motor control has the possibility of CAN communication, and therefore the measurement of vital physical quantities such as input and output voltage, current and power, as well as converter and motor temperatures, motor rotational speed, etc. Additionally, a battery charger as well as a battery management system (BMS) has also the possibility of CAN communication. It measures temperature and voltage for each battery cell, as well as the voltage and current of the whole battery pack. A plan is to obtain a power converter for a solar panel that would also use CAN to communicate with the vehicle systems. A torque sensor, together with the rotational speed measurement, gives mechanical power and is also essential for research. In the first step those physical quantities will be measured via a small data 




Figure 5. PLC based measurement system.

acquisition system connected to a PC. A low cost National Instruments NI USB-6009 data acquisition device will be used for the AD conversion. This DAQ will measure $12 \mathrm{VDC}$ consumption (voltage and current) as well as the ambient temperature. Such concept proves to be the cheapest and yet the most open system so it is chosen for installation.

\section{DEVELOPMENT OF MECHANICAL POWER MEASUREMENT SYSTEM}

Monitoring of motor torque and rotational speed in realtime provides a valuable insight of the vehicle behaviour and characteristics. The torque and rotational speed measurement subsystem was designed to accommodate the requirements for easy installation, low price, modularity and flexibility for various

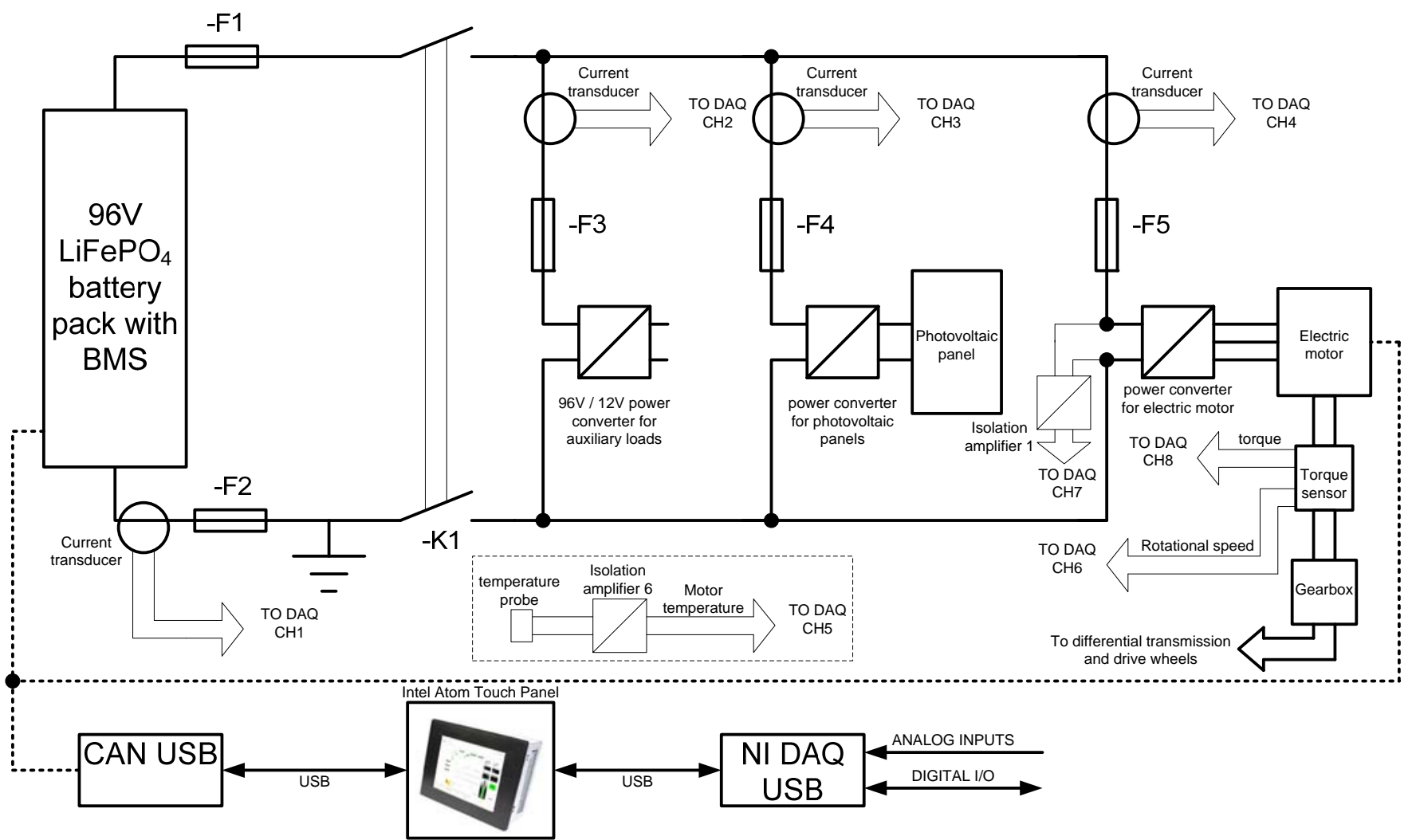

Figure 6. DAQ system based measurement system. 


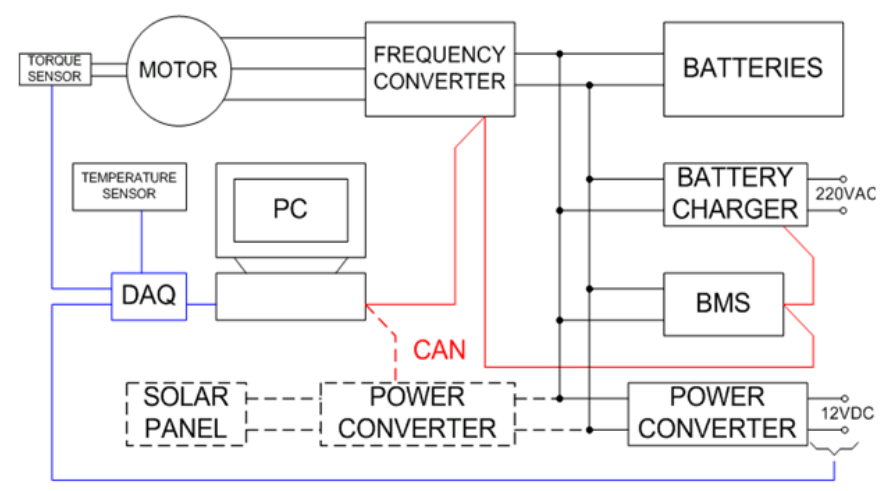

Figure 7. CAN based system.

test purposes. The block diagram of the measurement system is depicted in Fig. 8. The system consists of two main parts: the wireless torque measurement node, mounted directly on the cardan shaft, and the receiver unit which processes the measurement signals and transmits the results to the main processing unit of the vehicle. The receiver unit also has an input for interfacing with the rotational speed sensor.

Torque is measured directly by means of strain gauges mounted on the shaft in a full bridge configuration, which inherently provides high sensitivity and good temperature compensation [11]. The voltage $U$ measured on the bridge diagonal can be converted into torque $M$ as follows:

$$
M=\frac{\pi}{32} \cdot \frac{R_{t} \cdot G}{K_{t} \cdot R_{c}} \cdot \frac{\left(D^{4}-D_{u}^{4}\right)}{D} \cdot \frac{\left|U-U_{m 0}\right|}{\left|U_{c}-U_{m 0}\right|}
$$

where $R_{t}$ is the strain gauge resistance, $G$ the shear modulus $\left(\mathrm{N} / \mathrm{m}^{2}\right), K_{t}$ the gauge factor, $R_{c}$ a calibration resistance, $D$ the outer shaft diameter, $D_{U}$ the inner shaft diameter, $U_{c}$ the gain calibration voltage and $U_{m 0}$ the zero bridge voltage. Equation (1) enables torque calculations without the application of the reference momentum, under the assumption that all factors in (1) are known. This enables a simple two-step calibration procedure which is easy to automate: the zero calibration is performed in a first step (measurement of voltage $U_{m 0}$ with unloaded shaft when the calibration resistance $R_{c}$ is disconnected), while the gain is calibrated in a second step (measurement of voltage $U_{c}$ with unloaded shaft when calibration resistance $R_{c}$ is connected to simulate bridge unbalance under known load). Zero voltage $U_{m 0}$ represents the initial bridge unbalance due to the differences in the nominal resistance values of the four strain gages, as well as any residual

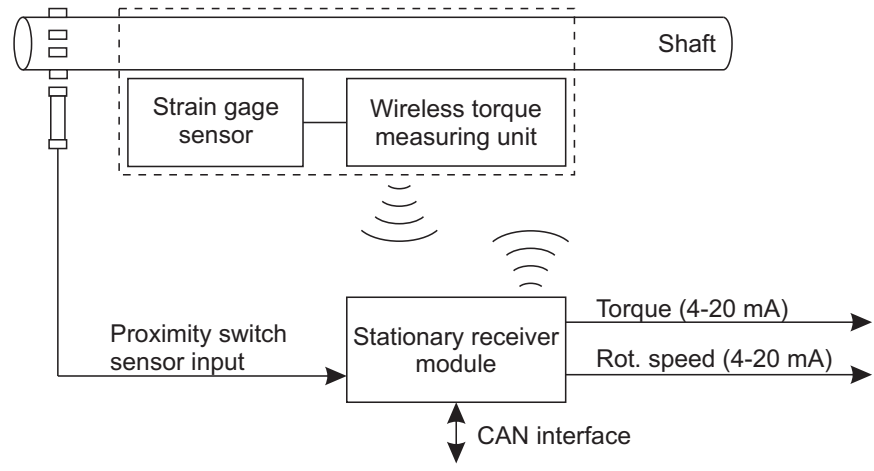

Figure 8. The block diagram of the torque and rotational speed measurement subsystem. strain. Alternatively, the torque measuring channel calibration can be performed by a two-point measurement and application of the reference momentum. The power can be calculated from the measured torque and the rotational speed by means of the known relation:

$$
P=2 \cdot \pi \cdot M \cdot n
$$

The block diagram of the shaft-mounted wireless torque measurement unit is depicted in Fig. 9. The module consists of a precision instrumentation amplifier, a high-resolution $\Delta \Sigma$ $\mathrm{A} / \mathrm{D}$ converter (ADC), a reference voltage source, a microcontroller, a radio-frequency $(\mathrm{RF})$ transceiver, a highefficiency switching-mode regulator, and the battery. Due to the oversampling operation principle of the ADC, a simple passive first-order low-pass antialiasing filter is sufficient. The sampling rate of the torque measurement channel is programmable up to $2 \mathrm{kS} / \mathrm{s}$. The microcontroller itself controls the sampling process, the data stream packaging and the two way RF communication through the $2.4 \mathrm{GHz}$ ISM RF link. The radio communication was implemented by using the XBee module that provides a simple transparent UART operation mode, without the need for developing complex dedicated packet oriented RF protocols. The data rate of $115.2 \mathrm{kbps}$ is sufficient for transmission of digitized torque samples to the stationary receiver unit. The total current consumption of the torque measurement module is less than $70 \mathrm{~mA}$ (with $350 \Omega$ strain gage), thus providing autonomous operation of about 30 hours under full load, assuming that the system is powered from four $2000 \mathrm{mAh}$ NiMH batteries. This figure can be further improved for practical purposes by driving the torque measurement module into the idle state when it is not in use. The vehicle control system can bring the torque measurement module into sleep mode with negligible power consumption by means of a two way RF communication link. The module can be woken up on demand by sending the appropriate control packet through the RF link. The ultra-low power operation in sleep mode with remote wake-up capability is achieved by means of a low duty cycle listening algorithm.

The receiver module contains a microcontroller, an XBee RF module, a rotational speed sensor input, analogue current loop outputs (4 to $20 \mathrm{~mA}$ ), a CAN interface, and the power supply. The microcontroller receives torque samples through the UART interface connected to the XBee module. The data stream is decoded in real time and the torque measurement signals are transmitted to the main vehicle control unit by means of the analogue current loop. An inductive proximity

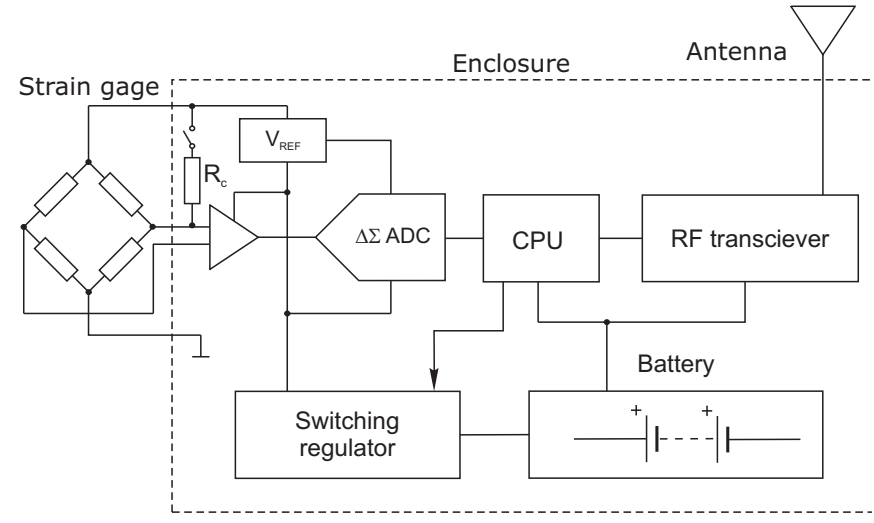

Figure 9. The block diagram of the wireless torque measurement unit mounted on shaft. 
switch is used to measure the rotational speed. The output of the rotational speed measurement sensor is connected directly to the microcontroller which performs high precision measurements by simultaneous measurement of time and frequency from the incoming pulses [12]. Both quantities are available as analogue current outputs 4-20 mA. Alternatively, these quantities can be transmitted through the CAN interface but the basic prototype implementation favoured analogue outputs due to software and hardware simplicity of the implementation. The CAN interface is also useful for sending control messages from the vehicle control unit for e.g. powering down or waking up the torque measurement module. The power management of the stationary receiving module is not an issue, because the power is supplied by the vehicle main power supply.

\section{MOBILE LABORATORY POSSIBILITIES}

The main idea is to establish a platform for research on different systems contained in a modern electric vehicle. Therefore an ordinary car with an internal combustion engine is transformed into an electric car with lots of equipment that enables the intended research.

At first, the car will be equipped with systems necessary for passing homologation and registration. Therefore it will be fully driveable in everyday traffic. Afterwards, additional equipment will be added in order to enhance research possibilities.

Several research goals are now planned. For example, the drive range detection will be investigated. In order to do that properly, one should know the battery model, the energy stored in the battery pack, the current energy consumption, etc. Since this system can be linked with maps (for example with Google maps via internet connection enabled by GPRS, EDGE or similar), the user could enter the desired destination and check if it is possible to reach this destination and under what circumstances (estimated arrival time, average velocity, etc). Also the user could see, using the same application, the reachable destinations such as hotels, towns, gas stations, etc. Of course, it would be possible to limit the vehicle speed in order to expand the drive range at users will.

Another research that will be achievable with this vehicle is the study of the usage of solar panels as alternative source for battery charging. For example, most of the cars are used for driving to work and back home. Since they stand still at a parking lot, solar panels installed on them can be used for battery charging thus reducing battery charging from the power grid. If there is a solar panel with an area of $2 \mathrm{~m}^{2}$ it can, in ideal conditions, produce a power of $300 \mathrm{~W}$. When this value is reduced to a more realistic level, $150 \mathrm{~W}$ average power can be taken into consideration. Therefore approximately $1200 \mathrm{Wh}$ of electric energy can be produced, or $11.7 \mathrm{Ah}$ for $102.4 \mathrm{~V}_{\mathrm{DC}}$. That is almost $12 \%$ of the vehicle battery pack capacity, and that is not something that can be ignored.

The efficiency of the different system parts can be measured and determined for different operating points thus enabling their optimal usage. In this research, the general idea is to increase the total efficiency in order to enhance the drive range (or to reduce the weight of the vehicle).

\section{CONCLUSION}

The presented system mounted on the described vehicle is capable of carrying out research on different problems such as optimal energy distribution, efficiency of separate parts as well as the whole vehicle, vehicle range prediction, etc. Since the vehicle will be used in everyday traffic, the obtained data will be closer to reality than in laboratory tests. Use of the CAN enables easy integration of the new equipment and the signals to the measurement and control systems of the vehicle. This system will also enable research on vehicle control strategies, e.g. optimization of the motor torque (vehicle acceleration) in order to safely reach the destination. This system will leave a lot of interesting research possibilities open as such vehicle actually represents a mobile laboratory for vehicle systems research.

\section{REFERENCES}

[1] X. Wang, T. Stuart, "Charge measurement circuit for electric vehicle batteries", IEEE Transactions on Aerospace and Electronic Systems, Volume: 38, Issue: 4, pp. 1201 - 1209, 2002, DOI 10.1109/TAES.2002.1145743

[2] A. Hande, S. Kamalasadan, A. Srivastava, "Selective Voltage Measurement System for Series Connected Battery Packs", Proceedings of the IEEE SoutheastCon, pp. 22 - 27, 2006, DOI 10.1109/second.2006.1629317

[3] T.P. Bohn, R.D. Lorenz, E.R. Olson, "Measurement of in-situ currents in a hybrid electric vehicle integrated power module using giant magnetoresistive sensors", Power Electronics in Transportation, pp. 55 - 59, 2004, DOI 10.1109/ PET.2004.1393795

[4] Yin Ying, Xin Tang, Xiaonan Li, Xiaodong Li, H. Weiss, "Implementation of Rugged Measurement System on Electric Vehicle", International Conference on Actual Problems of Electronic Instrument Engineering, pp. 166 - 171, 2006, 10.1109/APEIE.2006.4292413

[5] Yu Zhilong, Li Dongsheng, He Long, Wang Haiying, "Hardware design on electric vehicle LCD display system based on CAN bus", 6th International Forum on Strategic Technology (IFOST), Vol. 1, pp. 283 - 286, 2011, DOI 10.1109/ IFOST.2011.6021023

[6] H. Weiss, "Revitalization, performance measurement, and improvement of electric vehicles", International Conference on Actual Problems of Electronic Instrument Engineering, pp. 108 - 113, 2008, DOI 10.1109/APEIE.2008.4897068

[7] K. Muehlbauer, F. Bachl, D. Gerling, "Comparison of measurement and calculation of power losses in AC/DCconverter for electric vehicle drive", International Conference on Electrical Machines and Systems (ICEMS), pp. 1 - 4, 2011, DOI 10.1109/ICEMS.2011.6073652

[8] M. Gougani, M. Chapariha, J. Jatskevich, "Locking electric differential for brushless DC machine-based electric vehicle with independent wheel drives", IEEE Vehicle Power and Propulsion Conference (VPPC), pp. 1 - 6, 2011, DOI 10.1109/VPPC.2011.6043122

[9] L.W. Juang, P.J. Kollmeyer, T.M. Jahns, R.D. Lorenz, "System identification-based lead-acid battery online monitoring system for electric vehicles", IEEE Energy Conversion Congress and Exposition (ECCE), pp. 3903 - 3910, 2010, DOI 10.1109/ECCE.2010.5617776

[10] J.T.B.A. Kessels, B. Rosca, H.J. Bergveld, P.P.J. van den Bosch, "On-line battery identification for electric driving range prediction", IEEE Vehicle Power and Propulsion Conference (VPPC), pp. 1 - 6, 2011, DOI 10.1109/VPPC.2011.6043022

[11] I. J. Garshelis, "Torque and Power Measurement", in Measurement, Instrumentation, and Sensors Handbook CRCnetBase 1999, CRC Press LLC, 1999.

[12] H. Dzapo, Z. Stare and N. Bobanac, "Portable Wireless Measuring System for Monitoring Motor Shaft Parameters", in Proc. 21th IEEE Instrumentation and Measurement Technology Conference, Como, Italy, 2004, pp. 901-906. 